\title{
TWO MAPPINGS IN CONNECTION TO FEJÉR INEQUALITY WITH APPLICATIONS
}

\section{Rostamian Delavar And S. S. DRAgomir}

Abstract. By the use of two $h$-convex mappings $H_{g}$ and $F_{g}$, some results and refinements related to the $h$-convex version of Fejér inequality are established. Also some applications for obtained inequalities in connection with Beta function of Euler are given.

Mathematics subject classification (2010): 26D15, 26A51, 52A01.

Keywords and phrases: $h$-convex function, Fejér inequality.

\section{REFERENCES}

[1] M. Bombardelli And S. Varošanec, Properties of h-convex functions related to the HermiteHadamard-Fejér inequalities, Comput. Math. Appl. 58 (2009), 1869-1877.

[2] W. W. BRECKNER, Stetigkeitsaussagen für eine Klasse verallgemeinerter konvexer funktionen in topologischen linearen Räumen, Publ. Inst. Math. 23 (1978), 13-20.

[3] S. S. Dragomir, A mapping in connection to Hadamard's inequalities, An. Öster. Akad. Wiss. Math.-Natur., (Wien), 128 (1991), 17-20.

[4] S. S. Dragomir, On Hadamard's inequalities for convex functions, Mat. Balkanica 6 (1992), 215222.

[5] S. S. Dragomir, Two Mappings in Connection to Hadamard's Inequalities, J. Math. Anal. Appl. 167 (1992), 49-56.

[6] S. S. Dragomir and C. E. M. Pearce, Selected topics on Hermite-Hadamard inequalities and applications, RGMIA Monographs, Victoria University, 2000, (online: http://ajmaa.org/RGMIA/ monographs.php/).

[7] S. S. Dragomir, J. PeČarić, L. E. Persson, Some inequalities of Hadamard type, Soochow J. Math. 21 (1995), 335-341.

[8] L. FEJÉR, Über die fourierreihen, II, Math. Naturwise. Anz Ungar. Akad. Wiss. 24 (1906), 369-390.

[9] E. K. Godunova, V. I. Levin, Neravenstva dlja funkcii širokogo klassa, soderžaščego vypuklye, monotonnye i neko-torye drugie vidy funkcii, in: Vyčislitel. Mat. i. Mat. Fiz. Mežvuzov. Sb. Nauč. Trudov, pp. 138-142, MGPI, Moskva, 1985.

[10] B. Micherda ANd T. RAJBA, On Some Hermite-Hadamard-Fejér inequalities for $(k, h)$-convex function, Math. Inequal. Appl. 4 (2012), 931-940.

[11] J. PARK, Inequalities of Hermite-Hadamard-Fejér type for convex functions via fractional integrals, Int. J. Math. Anal. 8, 59 (2014), 2927-2937.

[12] A. W. Robert and D. E. Varbeg, Convex functions, Academic Press, 1973.

[13] M. Rostamian Delavar And M. De La Sen, On Generalization of Fejér Type Inequalities, Communications in Mathematics and Applications, 8, 1 (2017), 31-43.

[14] M. Z. SariKaYa, On new Hermite Hadamard Fejér type integral inequalities, Stud. Univ. BabesBolyai Math. 57, 3 (2012), 377-386.

[15] K.-L. Tseng, G.-S. YAng And K.-C. Hsu, Some Inequalities for Differentiable Mappings and Applications to Fejér Inequality and Weighted Trapezoidal Formula, Taiwanese J. Math. 15, 4 (2011), $1737-1747$.

[16] S. VarošAnec, On h-convexity, J. Math. Anal. Appl. 326 (2007), 303-311. 